followed by by extended oligoarthritis $(27 \% / 26 \%)$, polyarticular psoriatic arthritis $(18 \% / 16 \%)$ and RF positive polyarthritis $(5 \% / 8 \%)$. Disease duration $(2.3+/-3.0$ vs $1.9+/-2.7$ was statistically higher in the oral cohort $(p=0.04)$ but age at onset and baseline were similar. The baseline disease activity was higher in the s.c. cohort (JADAS10 16.5+/-7.2 compared to 14.7+/-8.2; $p<0.001$ and active joint count $9.0+/-10.1$ vs. $7.4+/-7.7 ; p=0,011$ ). The weekly MTX dosages were comparable with $13.6+/-5.4 \mathrm{mg}$ and $13.3+/-4.5 \mathrm{mg}$. Concomitant treatment with NSAIDS $(95 \% / 89 \%)$, oral steroids $(24 \% / 25 \%)$ or intraarticular steroids $(6 \% / 8 \%)$ were comparable.

After 12 months of treatment, $150(38.3 \%)$ reached a JIA ACR90 with oral MTX and $131(35 \%)$ with s.c MTX while $86(21.8 \%)$ and $72(19.5 \%)$ reached JADAS-remission (JADAS10 1 ). By Kaplan-Meyer- analysis no difference in the early kinetic of response was found. Upon total observation for up to 7.5 years in the intention to treat population (patients discontinuing MTX due to inefficacy or intolerance or starting a biologic were calculated as non-responders) more patients in the oral cohort reached a JADAS-remission (162; $41 \%)$ than with s.c MTX $(126 ; 34 \%)$ which was stastically borderline significant $(p=0,05$; odd's ratio $1.2[95 \mathrm{Cl} 1.0-1.8])$

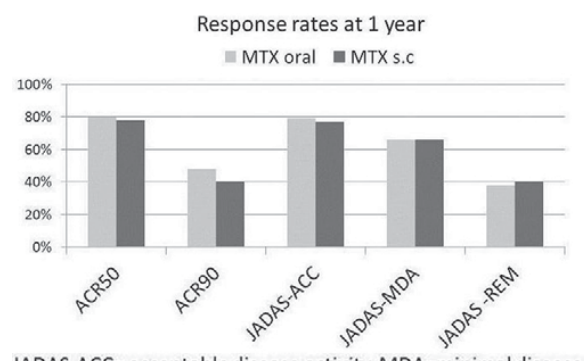

JADAS-ACC $=$ acceptable disease activity, MDA=minimal disease activity, Rem=remission

Conclusions: Data from the BIKER registry out of the clinical practice do show a high rate of JIA patients reaching a significant JIA-ACR response as well as JADAS-remission upon MTX as a sole DMARD. However, on the long term more patients with oral MTX reached JADAS remission. By Kaplan Meyer analyses we did not observe a superiority of s.c. MTX in the kinetic of response. The limitations of our analysis lie the character of a registry study, the lack of randomisation and study protocol leaving all decisions to start or to stop MTX by the responsible rheumatologist. Thus such data are preliminary and should be confirmed by randomized studies.

Disclosure of Interest: None declared

DOI: 10.1136/annrheumdis-2017-eular.3530

\section{THU0530 CHARACTERIZATION OF A COHORT OF PSORIATIC JUVENILE IDIOPATHIC ARTHRITIS PATIENTS FROM A PAEDIATRIC UNIVERSITY HOSPITAL IN SPAIN}

S. Hernández-Baldizón ${ }^{1}$, A. Zacarías-Crovato ${ }^{2}$, V. Torrente-Segarra ${ }^{3}$, J. Calzada-Hernández ${ }^{2}$, J. Sanchez Manubens ${ }^{2}$, E. Iglesias ${ }^{2}$, R. Bou Torrent ${ }^{2}$,

J. Anton $2 .{ }^{1}$ Rheumatology, Juaneda Miramar Hospital, Palma de Mallorca;

${ }^{2}$ Paediatric Rheumatology Unit, Sant Joan de Deu University Hospital,

(Esplugues)Barcelona; ${ }^{3}$ Rheumatology, Moises Broggi General Hospital, Hospitalet de Llobregat, Spain

Background: Juvenile Psoriatic Arthritis (JPsoA) is a subtype of Juvenile Idiopathic Arthritis (JIA) present in 7\% of JIA patients (1). Psoriasis is present in $0.5-1 \%$ of children. Diagnosis is often difficult, with the articular manifestations often preceding skin disease by years. Data is scarce in the Spanish population. Objectives: Describe demographical and clinical caracteristics of our cohort of JPsoA.

Methods: Descriptive, transversal study of patients attended from 1/201212/2016. Included were all complyers with ILAR Criteria (2) for JPsoA (Edmonton 2001). We also included the Wallace Criteria for clinical inactive disease (3) as a variable and endpoint. Data were included and analyzed using SPSS MAC 20.

Results: 31 patients were included: $18(59 \%)$ girls, $13(41 \%)$ boys. All Caucasian. They comprised $5 \%$ of all our JIA patients in that period.

Mean age at diagnosis was 7.4 years. All were RF-; 9 (29\%) ANA+; $4(12.9 \%)$ HLA-B27+; articular onset $17(55 \%)$ and cutaneous onset $14(45 \%) .9(29 \%)$ had Temporomandibular Joint (TMJ) symptoms: $5(16 \%)$ had pain and $4(12.9 \%)$ had a positive MRI for TMJ synovitis.

Plaque psoriasis $14(45 \%)$, guttata $3(9.6 \%)$ and $3(9.6 \%)$ had both. Dactylitis 8 (26.6\%); enthesitis $6(19.35 \%)$. Joint disease was mainly oligoarticular $15(48 \%)$, monoarticular $14(45 \%)$ and polyarticular $1(3.2 \%)$. Axial disease $4(12.9 \%)$ at follow-up. 7 (22.5\%) uveitis; 5 (77\%) were ANA +. 3 (9.6\%) onicodistrophia and 6 (19.3\%) enthesitis.

All patients received NSAIDs; $30(96 \%)$ methotrexate; 6 (19.3\%) switched to leflunomide. $16(51.6 \%)$ received biologic treatment and $9(29 \%)$ more than one. Articular debutants $10(32.2 \%)$ received biologic treatment more than the those with cutaneous onset $6(19.35 \%)$. We report $3(9.6 \%)$ anti-TNF/paradoxal psoriasis events.

Wallace Inactivity Criteria were achieved in $25(80.6 \%)$, with no differences between the biologic and DMARDS groups in time up to Achieving Wallace
Criteria (TimeWall). TMJ positive MRI did have a negative effect on TimeWall with $2(66.6 \%) \geq 8 \mathrm{yr}(8,11 \mathrm{y})$ to TimeWall.

Conclusions: We describe the clinical features and demographics of a series of spanish JPsoA patients. We found more oligoarticular and monoarticular involvement and an important presence of enthesitis and dactylitis; higher frecuency of uveitis than published data $(22.5 \%$ vs. $10-15 \%)$. Some were ANA-, reinforcing the need for screening. More than half required biologic treatment, and several cases we needed to switch drugs. Almost $60 \%$ of the patients were girls. Articular onset was associated with more active, harder to treat disease. TMJ positive RMI was associated with longer TimeWall. However, Wallace Criteria were not achieved globally.

References:

[1] T.R.Southwood, R.E. Petty, P.N. Malleson, et al. Psoriatic arthritis in children, Arthritis Rheum. 32 (1989) 1007-1013.

[2] Petty RE, Southwood TR, Manners P, et al. ILAR Classification of Juvenile Idiopathic Arthritis: second revision, Edmonton 2001, J. Rheumatol. 31 (2004) 390-392.

[3] Wallace CA, Giannini GH, Et Al. American College of Rheumatology provisional criteria for defining clinical inactive disease in select categories of juvenile idiopathic arthritis. Arthritis Care Res (Hoboken). 2011 Jul;63(7):929-36.

Disclosure of Interest: None declared

DOI: 10.1136/annrheumdis-2017-eular.6645

\section{THU0531 USE OF RITUXIMAB IN PAEDIATRIC RHEUMATOLOGY - EXPERIENCES FROM A SINGLE TERTIARY CENTRE}

S. Deepak $^{1}$, R. Obrien ${ }^{2}$, K. Warrier ${ }^{1}$, E. Mcdermott ${ }^{2}$, S. Rangaraj ${ }^{1} .{ }^{1}$ Paediatric rheumatology, Nottingham Childrens hospital; ${ }^{2}$ Immunology, Nottingham university Hospitals NHS Trust, Nottingham, United Kingdom

Background: Rituximab is an anti-CD20 monoclonal antibody therapy used widely in the management of paediatric rheumatological conditions. Studies suggest that Rituximab is safe and effective in rheumatic autoimmune diseases, but data on paediatric use remains limited. Although Rituximab spares plasma cells, hypogammaglobulinaemia can still develop, leading to recurrent infections. Frequency of hypogammaglobulinaemia in children receiving Rituximab for rheumatological conditions is unknown.

Objectives: To analyse the use of Rituximab in a tertiary Paediatric Rheumatology centre over the last 15 years (2001-2015). The primary aims were to identify the number of patients who received Rituximab, the underlying diagnoses and the response to treatment. Our secondary aims were to identify the incidence of hypogammaglobulinemia associated with Rituximab use and the frequency and severity of infections. Frequency of monitoring of immunoglobulin levels, lymphocyte subsets and functional antibodies to pneumococcus were noted. Methods: Retrospective analysis of case notes, electronic records and laboratory data of patients who received Rituximab in the paediatric rheumatology department from 2001-2015.

Results: A total of 22 patients received Rituximab (total of $1500 \mathrm{mg} / \mathrm{m}^{2}$ per cycle over $2-4$ divided doses) during the study period. 3 were excluded due to insufficient data. Median time of commencement of Rituximab from diagnosis was 2 years 8 months. Of these, 12 patients achieved remission within 6 to 12 months. Rituximab was discontinued in the non-responders at 12 months.

\begin{tabular}{lrccc}
\hline Diagnosis & \multicolumn{2}{c}{ No of patients } & Median No of cycles & Remission achieved \\
\hline Polyarticular JIA & RF + & 2 & 3.5 & $2(100 \%)$ \\
& RF - & 4 & & 0 \\
JDM & 6 & 3 & $5(83 \%)$ \\
SLE & 5 & 2 & $4(80 \%)$ \\
Vasculitis (GPA) & 2 & 2.5 & $1(50 \%)$ \\
\hline
\end{tabular}

8 patients $(42 \%)$ were noted to have hypogammaglobulinaemia at some point. The role of cyclophosphamide contributing to hypogammaglobulinaemia could not be excluded in 2 and a further patient is currently being investigated for an underlying primary immune deficiency. In the remaining $5(26 \%)$ patients, we believe the low IgG levels are secondary to Rituximab, of which two needed long term Ig replacement. Overall 12 patients reported recurrent/severe infections of which 6 had low immunoglobulin levels.

Conclusions: RF+ JIA patients appear to have responded the best to Rituximab and RF- JIA patients the least (0/4), with good results in JDM and SLE subgroups $(80-83 \%)$. The incidence of hypogammaglobulinaemia secondary to Rituximab in our cohort was $26 \%$, which can be prolonged and worsen with increased number of cycles. Prior treatment with cyclophosphamide may be contributory. We suggest regular monitoring of immunoglobulin levels and lymphocyte markers on all patients prior to commencement of Rituximab and regular intervals subsequently, including further cycles.

Disclosure of Interest: None declared

DOI: 10.1136/annrheumdis-2017-eular.3311 